\title{
Sozioökonomische und politische Umbrüche in Südostasien Einführung zum Themenheft
}

\section{Frauke Kraas, Köln}

Der Großraum Ost- und Südostasien gehört heute - sieben Jahre nach Einsetzen der sog. Asienkrise wieder zu den dynamischsten Weltwirtschaftsregionen, auch wenn wachstumseuphorische Prognosen der späten 1980er und 1990er Jahre in Südostasien inzwischen gemäßigteren Beurteilungen gewichen sind und sich die Kernräume wirtschaftlichen Wachstums auf das ostasiatische Festland verlagert haben. Zweifellos konnte sich der Großraum damit als dritte Schlüsselregion der Weltwirtschaft etablieren, weshalb die Epoche einseitiger Dominanz des sog. Westens auch in wirtschaftlicher Hinsicht zur Neige geht. Absehbar ist ferner, dass sich angesichts wachsender welt- und regionalwirtschaftlicher Verflechtungsprozesse verkürzte Wachstumszyklen, geringere Planungssicherheit und größere Anfälligkeit gesamtgesellschaftlicher Prozesse gegenüber globalen und regionalen sozioökonomischen wie politischen Einflussfaktoren einstellen werden.

Mit dem zweifachen ökonomischen Umbruch in Ostund Südostasien - unerwarteter Wirtschaftsboom seit Mitte der 1980er Jahre sowie plötzlich und unerwartet einsetzende Wirtschaftskrise im Jahr 1997 - gehen auch einschneidende soziale und politische Strukturumbrüche einher, deren Folgen erst mit Zeitverzögerung und innerhalb der Staaten raum-zeitlich stark differenziert sichtbar werden. Bei dem Versuch einer Bilanz für Südostasien nach zwei Jahrzehnten Wirtschaftsboom und -krise lässt sich ein insgesamt bemerkenswertes Gesamtbild mit folgenden zentralen Charakteristika festhalten, bei denen der zweifache wirtschaftliche Umbruch jeweils eigene Zeichen setzte.

(1) Hinsichtlich der demographischen Entwicklung im Großraum lassen sich vier grundsätzlich veränderte Entwicklungstrends der letzten Jahrzehnte beobachten, die allerdings nur teilweise mit dem sozioökonomischen Entwicklungsgang korrelieren.

- Nach drei Jahrzehnten allgemein starken Bevölkerungswachstums nach dem Zweiten Weltkrieg ist in den meisten Staaten (allen voran Thailand, aber auch Indonesien und Vietnam) eine deutliche Verlangsamung der Zuwachsraten zu beobachten. In diesen Staaten beginnt man sich Gedanken über die Konsequenzen eines zukünftig veränderten Altersaufbaus für die Sozial- und Versorgungssysteme zu machen.

- Auf hohem, wenn auch sinkendem Wachstumsni- veau stehen Entwicklungsländer wie Kambodscha (mit einem durchschnittlichen jährlichen Bevölkerungswachstum von 3,7\%) oder Laos (>2,5\%), aber auch in Staaten mit pronatalistischen Bevölkerungspolitiken, darunter Brunei (2,7\%), Singapur (2,7\%) und Malaysia (2,4\%; alle Angaben: 1995-2000; Association of Southeast Asian Nations (ASEAN) 2002). In diesen Staaten zeitigten wirtschaftlicher Boom und Krise kaum Auswirkungen auf die Entwicklungen.

- Unter dem Einfluss der Wirtschaftsumbrüche veränderten sich die innerstaatlichen und internationalen Migrationsprozesse: Die Land-Stadt-Wanderungen vervielfachten und differenzierten sich in Volumen und Dynamik, hinzu kam eine erhebliche internationale Wanderung ungelernter, aber auch qualifizierter Arbeitskräfte in die boomenden Volkswirtschaften (HUSA \& WOHLSCHLÄGL 2000).

- In allen Staaten Südostasiens erfolgen rapide Urbanisierungsprozesse. So stieg die Urbanisierungsquote in Südostasien insgesamt von 14,8\% (1950) über $24,3 \%$ (1980) auf $37,5 \%(2000)$, in Indonesien von $12,4 \%$ über $22,2 \%$ auf $41,0 \%$, in den Philippinen von $27,1 \%$ über $37,5 \%$ auf $58,6 \%$ und in Malaysia von $20,4 \%$ über $42,0 \%$ auf $57,4 \%$ (UNITED Nations (UN) 2002: 28/29). Damit einher gingen ein Bedeutungsgewinn der urbanen gegenüber den nationalen Ökonomien und breite Ausdifferenzierungen des formellen wie informellen Sektors in den Städten. Beschleunigte Prozesse massiver Flächenexpansion und innerurbanen Strukturwandels führen $\mathrm{zu}$ strukturellem und funktionalem Umbau sowie vielschichtigen sozialen Verdrängungsprozessen in den (vor allem: Haupt-) Städten und Metropolen.

(2) Boomphase und sog. Asienkrise prägten - ebenfalls insbesondere in den urbanen Regionen - die wirtschaftlichen Entwicklungen in mehrfacher Hinsicht:

- Bruttoinlandsprodukt (BIP) und durchschnittliches Pro-Kopf-Einkommen haben sich in allen Staaten Südostasiens in der Zeit zwischen Mitte der 80er Jahre und 1997 mindestens verdoppelt (z.B. Singapur: 20.847 US\$, Kambodscha 270 US\$ durchschnittliches Pro-Kopf-Einkommen, 2001; ASEAN 2002) - wenngleich mit gravierenden zwischen- wie innerstaatlichen Disparitäten. Bis heute konnte allerdings noch kein Staat die Niveaus aus der Zeit vor Einsetzen der sog. Asienkrise zurückerlangen. Steigender Wohlstand ist sichtbar z.B. am wachsenden Mechanisierungsgrad in der Landwirtschaft, einer Vielzahl moderner Hochhäuser, erheblich gestie- 
gener Mobilität sowie hoher Nachfrage nach Konsumgütern inklusive Luxuskonsum.

- In allen Staaten sank die Bedeutung des primären Sektors von Agrar- und Forstwirtschaft sowie Fischerei (Minimalwerte von $8,0 \%$ des BIP-Anteils in Malaysia und $10,0 \%$ in Thailand, Maximalwert bei $52,1 \%$ in Laos, ohne Berücksichtigung der Stadtstaaten; ASEAN 2002). Dies darf nicht über die nach wie vor enorme Bedeutung der Landwirtschaft für Beschäftigung und Nahrungssicherung hinwegtäuschen (was nicht für Singapur, Brunei und Malaysia gilt). Auffällig ist die starke Ausweitung von Fischfang und Aquakulturen (vor allem in Thailand; Uthoff 1991) - inzwischen selbst in Kambodscha und Myanmar/Birma.

- In allen Staaten Südostasiens - wenn auch in unterschiedlicher Höhe - stiegen die Anteile der Verarbeitenden Industrie an der Entstehung des Bruttoinlandsprodukts (BIP): in Indonesien von $22,2 \%$ auf ca. $43,5 \%$, in Malaysia von $28,2 \%$ auf $44,9 \%$, in Thailand von $25,9 \%$ auf $44,3 \%$, bezogen auf 1990 und 2001 (Ostasiatischer Verein (OAV) 2002; ASEAN 2002). Von zentraler Bedeutung sind neben den Auslandsdirektinvestitionen (1997 noch 27,3 Mrd. US\$, 2000 nur noch 10,3 Mrd. US\$; ASEAN 2002) und steigenden Export- wie Importraten auch die jeweiligen Schlüsselindustrien, darunter die arbeitsintensive Textilindustrie (in Vietnam, Laos und Kambodscha), eigene Fahrzeugindustrien (z.B. Motorradproduktion in Thailand, Etablierung der eigenen Automarke Proton in Malaysia) sowie innovative Industriebereiche der Bio-, Computer- und Informationstechnologie (vor allem in Singapur und Malaysia).

- Die Anteile der Dienstleistungen am BIP stiegen kontinuierlich (Spitzenwert Singapur: 65,4\%, gefolgt von Malaysia: $47,1 \%$, am schwächsten in Kambodscha mit 36,4\%; ASEAN 2002), wobei in Singapur die quartären, in den Entwicklungsländern die niedrigen Dienstleistungen dominieren. Hohe und kontinuierlich wachsende Beiträge leistet der Tourismus: Allein zwischen 1995 und 2000 stiegen die Zahlen der Touristen um 27,1\% auf 37,8 Mio. Ankünfte; 10,2 Mio. reisten nach Malaysia, 9,5 Mio. nach Thailand. Dabei stammen die Touristen zu erheblichem Teil aus der ASEAN selbst (38,9\%) oder aus dem übrigen Asien (31,4\%); nur 14,2\% kommen aus Europa und $6,1 \%$ aus Amerika (1995-2000; ASEAN 2002).

- Ein massiver Ausbau der Verkehrs- und Kommunikationsinfrastruktur, besonders im Straßenbau und der Luftfahrt, erfolgte mit finanzieller Unterstützung durch internationale Entwicklungszusammenarbeit. Besonders Malaysia und Thailand entwickelten ausgedehnte Infrastrukturnetze. Die Transformationsstaaten erfuhren nach 30 Jahren Krieg und Bürgerkrieg (Kambodscha, Laos und Vietnam) bzw.
Selbst- und Fremdisolation (Myanmar/Birma) seit Beginn der 1990er Jahre einen enormen nachholenden Infrastrukturausbau, durch den periphere Landesteile erschlossen und an die zentralen wirtschaftlichen Aktivräume der einzelnen Staaten angebunden wurden.

- Nach dem Konkurs einer Vielzahl von Industrieunternehmen und Dienstleistungsbetrieben im Gefolge der sog. Asienkrise sowie im Zuge des beginnenden Abbaus von Personal in der Verwaltung stieg die Zahl der Arbeitslosen Schätzungen zufolge auf ca. $3,3 \%$ in Singapur und Thailand, 3,7\% in Malaysia, $5-10 \%$ in Brunei und Kambodscha, $11,2 \%$ in den Philippinen, $17 \%$ in Laos, mehr als $30 \%$ in Indonesien (Angaben für 2001; OAV 2002; Schätzungen differieren stark). Hinzu kommen die Zahlen saisonal unbeschäftigter und unterbeschäftigter Personen.

- Im Gefolge der Asienkrise nahm die Entsendung von Arbeitskräften ins Ausland zu: Hier dominieren die Philippinen mit - je nach Quelle - zwischen 3,0 und 1,1 Mio. sog. Überseearbeiter (2002, temporäre Migranten während der letzten fünf Jahre vor einer Zählung; National Statistical Office (NSO) 2003). In Thailand wurde die Zahl der Entsendungen, insbesondere in die USA, nach Japan und Israel, auf mehr als 200.000 (1999) angehoben (SüDOSTASIEN AKTUELL 3/1999: 185). Auch die kaum unmittelbar von der Krise betroffenen Staaten Laos, Kambodscha und Myanmar/ Birma werden indirekt dadurch einbezogen, dass viele vormals legal oder illegal in den Nachbarstaaten beschäftigte Personen, die dort vor der Wirtschaftskrise als billiger und nach Belieben einsetzoder abstoßbarer Arbeitskräftepuffer fungierten, seither nicht mehr gefragt sind und in ihre Heimatländer ausgewiesen werden.

(3) Der Versuch, die sozialen Folgen von Wirtschaftsboom und -krise zu umreißen und deren Ausmaß abzuschätzen, unterliegt zwei Schwierigkeiten: Zum einen werden die teils gravierenden sozialen Umbrüche erst mit Zeitverzögerung erkennbar; zudem sind sozialgruppenspezifische und regionale Differenzierungen bislang erst wenig erfasst und quantifiziert. Ferner ist Vorsicht bei der Deutung der Phänomene und Zahlenangaben insofern geboten, als soziale Veränderungen auf mehrdimensionale Ursachen zurückzuführen sind.

- Die Grund- und Spezialversorgung im Gesundheitsbereich verbesserte sich in allen südostasiatischen Staaten zumeist deutlich, allem voran in den Städten. Dies gilt besonders für Malaysia, Thailand, die Philippinen und Indonesien; eklatante Defizite bestehen unverändert in Laos, Kambodscha und Myanmar/Birma. Auch darf nicht verkannt werden, dass gerade die unteren Einkommensschichten kaum oder keinen Zugang zu adäquaten Gesund- 
heitsdienstleistungen besitzen - eine Situation, die durch Budgetkürzungen und Abbau staatlicher Subventionen seit der sog. Asienkrise verschärft wurde.

- Alle Staaten verstärkten ihre Bildungsanstrengungen erheblich; dies gilt in erster Linie dem Ausbau des allgemeinen Bildungswesens, in den entwikkelteren Volkswirtschaften (Singapur, Malaysia, Thailand) auch für die Bereiche der Sekundär- und beruflichen Weiterbildung. In Kambodscha und Vietnam dienen erhöhte Bildungsinvestitionen zugleich der Sicherung der politischen Einflussnahme durch die Regierungen.

- Mit wachsendem Wohlstand in der Zeit des Wirtschaftsbooms erhöhte sich die Zahl der Mittelschichtangehörigen bzw. es entstanden neue - ökonomische, nicht aber zugleich auch bildungsgetragene - Mittelschichten, mit jedoch allenfalls beginnender Übernahme von Verantwortlichkeit im sozialen Gefüge.

- Sowohl während der wirtschaftlichen Boomphase als auch seit Beginn der sog. Asienkrise stiegen die regionalen sozioökonomischen Disparitäten zwischen den sowie innerhalb der Staaten.

- Die sozialen Folgen der Wirtschaftskrise gehen zumeist direkt auf die sinkende Nachfrage nach Arbeit - einschließlich Arbeitslosigkeit, Unterbeschäftigung und fallender Löhne - sowie auf Ausgaben- und Preisveränderungen in Schlüsselbereichen zurück. Ursache hierfür sind sinkende Staatsausgaben für soziale Aufwendungen und Unterstützungsprogramme sowie eine erhebliche Teuerung der wichtigsten Grundnahrungsmittel. Beispielsweise stiegen in Thailand nach 1997 die Preise für Reis, Speiseöl und Zucker um bis zu $45 \%$; Elektrizität, Wasser und Benzin sowie Medikamente wurden um bis zu 18\% teurer (WorLd BANK 1999: 8/9).

- Nach erheblich gesunkenen Anteilen besonders städtischer Armer an der Gesamtbevölkerung während der Boomphase nahmen Armut und Unterversorgung, speziell von Kindern, behinderten und alten Menschen in den ländlichen Provinzen, aber auch unterer Sozialschichten in den Städten seit Einsetzen der sog. Asienkrise erneut stark zu. Die Werte variieren innerhalb der elf südostasiatischen Staaten enorm: Während die Anteile der Bevölkerung unterhalb der Armutsgrenze in Malaysia bei 8,6\% und in Thailand bei $12,9 \%$ liegen, erreichen sie in Kambodscha, Laos und Vietnam ca. $38 \%$, in den Philippinen gar 40\% (Angaben für unterschiedliche Erhebungsjahre zwischen 1998 und 2000; ASEAN 2002). Auch zeigen sich große zeitliche Schwankungen: Die Zahl der unterhalb der Armutsgrenze lebenden Thailänder etwa war von ca. $33 \%$ (1988) über 23\% (1992) auf 6,8 Mio. (11,4\%; Ende 1996) gefallen und stieg infolge der Wirtschaftskrise sprunghaft wieder auf etwa 7,9 Mio. an (ca. 13\%; Mitte 1998; World Bank 1999:3).
- Eindeutig krisenbedingt sind allgemein gestiegene Kriminalitätsraten, zunehmender Drogenkonsum und -handel, auch durch Kinder; die Zahl der Fälle von Drogendelikten stieg.

(4) Zumeist unterstützt durch die enorme wirtschaftliche Entwicklung vollzogen sich wesentliche, aber höchst unterschiedliche politische Entwicklungen in den Staaten Südostasiens.

- Dabei sind unterschiedlich verlaufende Prozesse politischer Diversifizierung zu beobachten, zu denen die Herausbildung und Verstärkung ethnischer Politisierung, teils Instrumentalisierung (z.B. in Indonesien, Vietnam und den Philippinen;SCHNEIDER 2000), das Entstehen und Erstarken von Nicht-Regierungsorganisationen (vor allem Thailand, aber auch Philippinen und Indonesien) sowie die Verbreiterung zivilgesellschaftlicher Organisationen und partizipativer Planungsansätze (z.B. Thailand und Indonesien) zählen. Das ökonomische Wachstum hat indes nur teilweise auch zur Intensivierung der politischen Diskussion oder gar Demokratisierungsprozessen innerhalb der Staaten geführt (insbesondere in Thailand, geringer in Indonesien und den Philippinen) - zumeist gereichte die Phase des Wirtschaftsboom den politischen Machthabern zur Legitimation und Festigung der eigenen Herrschaft (so etwa in Singapur, Malaysia, Vietnam und Kambodscha). Auch die Regierungen der Transformationsstaaten (Kambodscha, Laos, Vietnam und in gewisser Hinsicht auch Myanmar/Birma) benutzten die Einführung von Formen der Marktwirtschaft, denen anders als in Ost- und Ostmitteleuropa keine auch politische Transformation zur Seite stand, zur Stärkung der eigenen Machtposition.

- Zu Boomzeiten bereits - mit Ausnahme Thailands - oft enge Spielräume der öffentlichen Information und Pressefreiheit wurden seit Einsetzen der sog. Asienkrise wieder bzw. weiter beschnitten; kritische Analysen und Diskussion politischer Alternativen finden wenig Eingang in die Berichterstattung.

- In denjenigen Staaten, die krisenbedingt hohe Auslandskredite oder verstärkte Entwicklungshilfe erhalten (z.B. Indonesien, Kambodscha und Thailand), vollzieht sich in Strukturreformprogrammen unter dem externen Druck der Gebernationen und -organisationen eine erhebliche Restrukturierung, Reorganisation, teils auch Dezentralisierung von Verwaltungen, Finanzsektor, privaten und staatlichen Organisationen.

- Auffällig ist ferner die zunehmende Einbindung Südostasiens in multinationale Kooperationen, die inzwischen deutlich über die bis zum Ende der bipolaren Weltordnung in den 1990er Jahren hinausgehende Konzentration auf vor allem sicherheitspolitische Aspekte reicht: Im Zuge der Modernisierung und Stärkung von ASEAN, Asia- 
Europe Meeting (ASEM) und Asia-Pacific Economic Cooperation (APEC) finden zunehmend Internationalisierungs- und Integrationsprozesse statt.

Beim Versuch, die Rahmenbedingungen zukünftiger Entwicklungen in Südostasien auszuloten, lassen sich drei zentrale Tendenzen erkennen:

(1) Das rapide Wirtschaftswachstum in Südostasien war zu einem erheblichen Teil nicht von den Kräften freier Marktwirtschaft, sondern von gezielten nationalen Wirtschafts- und Industrialisierungspolitiken gelenkt gewesen, deren Defizite durch Strukturreformen angegangen werden müssen. Der Tatsache, dass jedoch auch soziale Sicherheit integraler Bestandteil marktwirtschaftlicher Ökonomien ist, wird erst seit Einsetzen der sog. Asienkrise gewisse Aufmerksamkeit zuteil. Zuvor waren die Aspekte sozialer Sicherheit allein als Hemmfaktoren für Wirtschaftswachstum und Beschäftigung eingestuft worden. In verhaltener Neubewertung treten nun die Probleme fehlender sozialer Stabilität (darunter Arbeitslosigkeit, Armut, soziale Desintegration) verstärkt in das Blickfeld der politischen Führungen (ScHolz 1999). Konkrete Handlungsoptionen - wie der Aufbau von Sozialund Arbeitslosenversicherungen - gewinnen indes in Südostasien nur langsam an Kontur (Schramm 2002). Dabei wird es darauf ankommen, neben staatlichen Ordnungen auch individuelle und gesellschaftliche Initiativen zu stärken, d.h. eine sinnvolle Mischung staatlicher Wohlfahrtspolitik und privater Aktivitäten zu verfolgen.

(2) Mit zunehmender Globalisierung und dem Aufstieg neuer gesellschaftlicher Gruppen - vor allem: ökonomische Mittelschichten und Bildungseliten mit ausländischen Studienabschlüssen -, die sich nicht in traditionelle Klientelstrukturen einpassen, beginnen sich veränderte innerstaatliche Verantwortlichkeiten auszuprägen. Diese gilt es miteinander zu verknüpfen und in nationale Konstruktionen sozialer Sicherheit einzubinden.

(3) Mit Blick auf eine weitere wirtschaftliche Konsolidierung und (Rück-)Gewinnung der Wettbewerbsfähigkeit ist wichtig, dass die dringend nötigen Reformen der Verwaltungs-, Kontroll- und Steuerungsstrukturen, der Unternehmenskultur und des Managements sowie auch die Privatisierung unrentabler Staatsunternehmen nicht zu einem Zeitpunkt abgewürgt werden, zu dem zur Vollendung der Reformanstrengungen noch weitere Veränderungen durchgeführt werden müssen. Akute Gefahr besteht derzeit darin, dass die Wirtschaft bei nahezu stagnierendem Außenhandel offensichtlich wegen steigenden Binnenkonsums wächst. Dies könnte ein Zeichen dafür sein, dass die bubble economy, die 1997 zur Wirtschaftskrise führte, sich nun auf die Ebene der privaten Haushalte ausgeweitet hat - mit möglicherweise gefährlichen Folgen.

Vor dem Hintergrund der umrissenen sozioökonomischen und politischen Umbrüche in Südostasien konzen- trieren sich die Beiträge des vorliegenden Themenheftes auf ausgewählte Fragestellungen, denen bisher wenig Aufmerksamkeit zuteil wurde: Kiese und Revilla Diez untersuchen die regionalen Innovationspotentiale - oft als Engpassfaktor für die Entfaltung einer eigenständigen Wirtschaftsdynamik eingestuft. ZIMMER stellt die Frage nach den Charakteristika und Problemen der Konversion im geostrategisch neugeordneten Südostasien. Jüngste Urbanisierungsprozesse und urbane Entwicklungsoptionen werden im Beitrag von KraAs untersucht. Aufbauend darauf stellt sich im Aufsatz von Clausen die Frage nach den Effekten von Globalisierungsprozessen auf die Interaktion von Teilräumen und sozialen Netzwerken. Und abschliessend gehen Pfeffer und YongVANIT der Frage nach der Wirkung agrarkolonisatorischer Massnahmen in einem Peripherraum nach.

\section{Literatur}

Association of Southeast Asian Nations (ASEAN) (2002): ASEAN Statistical Yearbook 2001. - Jakarta.

HuSA, K. \& H. WoHLSCHLÄGL (2000): Aktuelle Tendenzen der internationalen Arbeitsmigration in Südostund Ostasien vor dem Hintergrund von Wirtschaftsboom und Asienkrise. - In: Husa, K., Parnreiter, C. \& I. STACHER (Hrsg): Internationale Migration. Die globale Herausforderung des 21. Jahrhunderts? - Historische Sozialkunde 17, Frankfurt: 247-279.

National Statistical Office (NSO) (2003): Survey on Overseas Filipinos. - Manila.

Ostasiatischer Verein (OAV) (2002): Wirtschaftshandbuch Asien Pazifik 2002/2003. - Hamburg.

SCHNEIDER, H. (2000): Kulturkampf oder Ressourcenkonflikt? Hintergründe des Regionalkonflikts im Süden der Philippinen. - In: Petermanns Geographische Mitteilungen 144, 4: 14-19.

Scholz, W. (1999): Economic Crisis, Employment and Social Protection. The Case of Southeast Asia. - In: Asien 70: 48-53.

Schramm, B. (2002): Der Aufbau eines staatlichen Systems sozialer Sicherung in Thailand. - In: Internationales Asienforum 33, 1-2: 47-65.

SÜDOSTASIEN AKTUELL 3/1999: 185.

United Nations (UN) (2002): World Urbanization Prospects. The 2001 Revision. - New York.

Uthoff, D. (1991): Entwicklungsphasen und aktuelle Probleme der thailändischen Seefischerei. - In: BRÜcKNER, H. \& U. RADTKE (Hrsg.): Von der Nordsee bis zum Indischen Ozean. - Stuttgart: Steiner Verlag: 221-235. WorLD BANK (1999): Thailand Social Monitor. - Bangkok.

\section{Zusammenfassung: Sozioökonomische und politische} Umbrüche in Südostasien - Einführung zum Themenheft Mit dem zweifachen ökonomischen Umbruch - Wirtschaftsboom seit Mitte der 1980er Jahre sowie Wirt- 
schaftskrise seit 1997-gehen einschneidende soziale und politische Strukturumbrüche in Südostasien einher: (1) Bei der demographischen Entwicklung sind eine deutliche Verlangsamung des Bevölkerungswachstums sowie veränderte innerstaatliche und internationale Migrationsprozesse zu beobachten. (2) Die wirtschaftlichen Entwicklungen zeigen erhebliche Steigerungen von Bruttoinlandsprodukt und Einkommen, steigende Anteile des sekundären und tertiären Sektors, massiven Ausbau der Verkehrs- und Kommunikationsinfrastruktur, seit 1997 zunehmende Arbeitslosenzahlen und Entsendung von Arbeitskräften ins Ausland. (3) Soziale Folgen sind erhebliche Verbesserungen im Gesundheits- und Bildungsbereich, erhöhte Zahl der Mittelschichtangehörigen, aber auch vertiefte sozioökonomische Disparitäten,Ausgaben- und Preisveränderungen in Schlüsselbereichen sowie steigende Armut und Kriminalitätsraten. (4) Parallel finden höchst unterschiedlich verlaufende Prozesse politischer Diversifizierung statt mit engen Spielräumen für öffentliche Information und Pressefreiheit, Restrukturierung in Verwaltung und Wirtschaft sowie zunehmender Einbindung Südostasiens in multinationale Kooperationen. Sozialer Sicherheit als integralem Bestandteil marktwirtschaftlicher Ökonomien wird erst seit Einsetzen der sog. Asienkrise Aufmerksamkeit zuteil. Wichtig ist, dass nötige Reformen der Verwaltungsstrukturen, Unternehmenskultur und bei der Privatisierung unrentabler Staatsunternehmen nicht frühzeitig abgewürgt werden.

\section{Summary: Socio-Economic and Political Transforma- tion in Southeast Asia - Introduction to this issue}

In Southeast Asia, the economic boom from the mid1980's onwards and the economic crisis since 1997 led to radical social and political structural changes: (1) As far as demographic development is concerned, a marked decline of the population growth rate, as well as changed migration processes within states and on an international level are to be observed. (2) Economic development shows considerable increases in gross national product and income, growth in sections of secondary and tertiary activity, extensive development of transport and communications infrastructure, and since 1997 rising unemployment figures and the dispatch of labour abroad. (3) Social consequences are on the one hand considerable improvement in health and education services and a growing middle class, but on the other hand also greater socio-economic disparities, changes in expenditure and price levels in key areas as well as growing poverty and crime rates. (4) Simultaneously, very different processes of political diversification are underway with limited scope for public information and freedom of the press, restructuring in administration and the economy as well as the increasing integration of Southeast Asia in multinational co-operations. Attention has only been paid to social security as an integral part of market economies since the beginning of the so- called Asian crisis. It is important that necessary reforms of administrative structures, business structures and in the privatisation of unprofitable state enterprises are not prematurely stalled.

\section{Résumé: Transformations socio-économiques et poli- tiques dans le Sud-Est asiatique - une introduction}

Avec la double rupture économique - «Boom» à partir du milieu des années 1980, crise depuis 1997 - le SudEst asiatique fait l'objet de ruptures structurelles dans le domaine social et politique: (1) En matière de développement démographique, on observe un ralentissement prononcé de la croissance de la population, de même que des changements intraétatiques et internationaux dans les processus migratoires. (2) Les indicateurs économiques révèlent une augmentation sensible du produit intérieur brut et du revenu, une part croissante des secteurs secondaire et tertiaire, un renforcement massif des infrastructures de transport et de communication, ainsi que, depuis 1997, une augmentation des taux de chômage et des départs d'actifs à l'étranger. (3) Les conséquences en sont des améliorations conséquentes en matière de santé et de formation, une augmentation du nombre de personnes appartenant aux couches moyennes de la population, mais aussi des disparités socio-économiques accentuées, des modifications de dépenses et de prix dans des domaines-clés, ainsi qu'une pauvreté et des taux de criminalité croissants. (4) Parallèlement, se déroulent des processus de diversification politique très différenciés, aux marges de manœuvre étroites en matière d'information publique et de liberté de presse, de restructuration administrative et économique, de même qu'en ce qui concerne l'intégration grandissante du Sud-Est asiatique aux coopérations internationales. L'attention s'est surtout portée sur la sécurité sociale, en tant que composante intégrale des économies de marché, depuis le début de la soi-disante crise asiatique. Il importe que les réformes nécessaires portant sur les structures administratives, la culture d'entreprise et la privatisation d'entreprises publiques non rentables ne soient pas interrompues prématurément.

\section{Didaktische Hinweise}

- Welches sind die zentralen Umbrüche in der wirtschaftlichen Entwicklung Südostasiens seit Wirtschaftsboom und -krise und welche Trends sind $\mathrm{zu}$ erwarten?

- Welche Vor- und Nachteile ergeben sich aus den durch Wirtschaftsumbruch erwachsenen sozialen Entwicklungen?

Prof. Dr. Frauke Kraas, Geographisches Institut der Universität zu Köln, Albertus-Magnus-Platz, D-50923 Köln. e-mail: f.kraas@uni-koeln.de 